\title{
Vehicle Speed Prediction Using a Combined Neural Network of Convolution and Gated Recurrent Unit With Attention
}

Xiaohong Jiao ( $\sim$ jiaoxh@ysu.edu.cn )

Yanshan University

Zhennan Wang

Yanshan University

Zhao Zhang

Yanshan University

\section{Research Article}

Keywords:

Posted Date: March 7th, 2022

DOl: https://doi.org/10.21203/rs.3.rs-1382945/v1

License: (c) (i) This work is licensed under a Creative Commons Attribution 4.0 International License.

Read Full License 


\title{
Vehicle Speed Prediction Using a Combined Neural Network of Convolution and Gated Recurrent Unit With Attention
}

\author{
Zhennan Wang ${ }^{1,2}$, Xiaohong Jiao ${ }^{1,2,}$, and Zhao Zhang ${ }^{1,2}$ \\ ${ }^{1}$ Engineering Research Center of the Ministry of Education for Intelligent Control System and Intelligent Equipment, \\ Yanshan University, Qinhuangdao, 066004, China \\ ${ }^{2}$ School of Electrical Engineering, Yanshan University, Qinhuangdao, 066004, China \\ *jiaoxh@ysu.edu.cn
}

\begin{abstract}
Vehicle velocity prediction can benefit widespread applications, such as optimal vehicle propulsion systems and advanced driver assistance systems. Dynamic changes in the traffic environment strongly influence vehicle speed prediction, making it hard to predict vehicle speed accurately. In contrast, advances in intelligent transportation systems and machine learning have made it possible to predict short-term vehicle speed accurately. In this regard, this paper presents a novel vehicle speed prediction algorithm using deep learning methodologies. A practical temporal and channel attention module (TCAM) is designed for a feed-forward convolution neural network (CNN) to enhance valuable information and suppress redundant information. At the same time, a gated recurrent unit (GRU) with an attention network is utilized, having the advantages of analyzing significant relationships among time-series data through its memory function. The two prediction algorithms consisting of the CNN embedded in TCAM and the GRU with attention mechanism are fused to improve vehicle speed prediction performance. Simulation experiments using IPG-Carmaker software validate better prediction performance of the proposed model than traditional and existing deep learning prediction methods.
\end{abstract}

With the development of new energy hybrid electric vehicles and autonomous intelligent vehicles, vehicle velocity prediction plays an increasingly important role in the field of such as energy management optimization control and advanced driver assistance systems (ADAS) ${ }^{1}$. Energy consumption for a hybrid electric vehicle can be significantly reduced if its future speed is known in advance and used into management optimal $\operatorname{control}^{2},{ }^{3}$. Drivers can pass a traffic intersection successfully without a stop with the help of vehicle speed prediction, which can also reduce the energy consumption ${ }^{4}$. Besides, vehicle speed prediction can be integrated into cooperative driving strategy ${ }^{5}$ to ensure driving safety based on velocity prediction for the autonomous vehicles which need to cooperate with the nearby vehicles. The applications of vehicle speed prediction are greatly affected by the prediction accuracy. Therefore, it is necessary to develop a reasonable and accurate method for predicting future driving speed profiles in the time domain.

\section{Literature review}

In recent years, there have been some studies on the methods of short-term vehicle speed prediction, which can be generally divided into two main categories: stochastic vehicle speed prediction model and deterministic vehicle speed prediction model ${ }^{6}$. In the stochastic model, the Markov chain (MC) is a representative case ${ }^{7}$. It uses a state transition matrix calculated by the number of transitions between each state and the current states for predicting future states. According to different information, the hidden Markov model is utilized to predict the vehicle speed ${ }^{8},{ }^{9}$. The accuracy of the MC prediction model can be increased by establishing a high-order model, but the size of the state transition matrix will expand exponentially, and it is still challenging to cover vehicle speed states ${ }^{2}$. The deterministic methodologies can be mainly categorized into parametric and nonparametric methods ${ }^{10}$. Parametric methods rely on pre-defined models, in which model parameters are obtained and calibrated with empirical data. The autoregressive moving average model (ARMA) is a typical case of parametric methods applying for vehicle speed prediction ${ }^{11},{ }^{12}$. Due to the difficulty of choosing the incredibly suitable model parameters according to experience, it restricts the accuracy of building a speed model by the parametric methods ${ }^{13}$. In comparison, the data-driven methods without pre-defined parametric utilize historical data to predict the future, and they are more suitable for the actual car driving process with high randomness. Among the nonparametric models, neural networks (NNs), which utilize historical data to forecast, are most attractive and widely exploited ${ }^{14}$. Due to its powerful nonlinear mapping capability and robustness, it has become one of the critical technologies in nonlinear system predictive modeling and optimization control. Meanwhile, speed predictions based 
on the NNs trained by historical traffic data are more reasonable for public services vehicles, like buses and cargo trucks, with relatively fixed operation routes. The vehicle speed data on relatively fixed and repeated routes can reflect the characteristics of the regular driving cycle; therefore, the offline learning prediction models such as NNs can have good performance in real-time because the offline well-trained models can perform well in most of their driving conditions ${ }^{15},{ }^{16}$.

For the past few years, a significant amount of research on vehicle speed prediction based on NNs has been conducted. In literature ${ }^{17}$, the trip condition prediction model based on back propagation neural network (BPNN) is utilized to obtain the vehicle speed trajectory. The short-term vehicle velocity predictor is developed to improve the controller performance based on radial basis function neural network (RBF-NN) in ${ }^{18}$. The long-short-term memory network (LSTM) and the gated recurrent unit (GRU) compared to the recurrent neural networks (RNNs) are well suited for processing time-series data because they can overcome the problem of vanishing gradients ${ }^{19},{ }^{20}$ and can learn more complex nonlinear sequences to complete more forecasting tasks ${ }^{21}, 22$. The prediction of short-term velocity over 1 to 10 -second prediction horizon by LSTM is used as an input into an optimal EMS derivation algorithm $\mathrm{in}^{23}$. Researchers are working on combining more than one algorithm to attain higher accuracy for forecasting vehicle velocity. $\mathrm{In}^{24},{ }^{25}$, the predicted vehicle speed obtained from $\mathrm{MC}$ with the characteristics of the following state of speed change, and the BP neural network to fitting and compensating for velocity prediction. An individual vehicle's speed is affected by the traffic condition. Consequently, the authors in ${ }^{26}$ propose a prediction method that predicts the average traffic speeds of road segments by using neural network models based on historical traffic data, and hidden Markov models (HMMs) are then utilized to present the statistical relationship between individual vehicle speeds and the traffic speed. The future speed sequences are estimated by inflated 3D inception LSTM network, which can use the historical speed and image information to improve the accuracy of speed prediction ${ }^{27}$. The CNN-based model has been widely studied in traffic flow velocity prediction ${ }^{28}$, and can also be applied to vehicle speed prediction. $\mathrm{CNN}$ is used to receive input data and extract important features of the data, and LSTM is used to receive the output of the CNN layer ${ }^{29}$. In summary, the fusion prediction models that supply robust and accurate predictions for vehicle driving conditions are better than the single model.

Motivated by the analysis above, this paper makes full of the excellent learning and nonlinear fitting ability of NNs to design a novel NN-based vehicle speed prediction model to improve the prediction accuracy further. The main contributions of this paper are summarized as follows:

- A practical temporal and channel attention module (TCAM) block is developed, which has identical mapping properties and improves the information flow.

- The designed TCAM is embedded into CNN to form a CNN-TCAM network frame to capture more critical information adaptively.

- A novel speed prediction model, the CGAM network, based on a combined convolution neural network and gated recurrent unit with attention mechanism, is established to fit better the vehicle driving information and complete the speed forecast task.

The remaining parts of the paper are organized as follows: Methodology section presents the design of the vehicle speed prediction model. Results section presents experimental results, including data collection, modeling results, and analysis.

\section{Methodology}

CGAM network. The proposed CGAM network for the vehicle speed prediction is outlined in Figure 1, which consists of two branches, one is composed of a classic CNN network embedded with the developed TCAM, named as CNN-TCAM, and the other is the GRU integrated with an attention mechanism, named as GRU-Attention.

In neural networks, the inputs and extracted features contain different types of information and different contributions to predicting the vehicle speed. However, the most simple CNN methods consider different types of information equally and lack flexible modulation ability in dealing with them, limiting the representational ability and fitting capacity. Therefore, simply increasing the depth or width of the simple CNN methods can hardly achieve better improvement. To address these issues, we propose a TCAM block which can enhance the feature representation responses by automatically exploring temporal and channel feature interdependencies. The ability of the recurrent neural network to capture the long-term dependence of time series is stronger than the convolutional network. Therefore, we use the GRU-Attention network to capture long-term dependency in a time series between data. The Dropout layer can reduce the complexity of the model, enhance the generalization ability of the model, and prevent overfitting. Also, an attention mechanism is utilized to strengthen pivotal information and weaken trivial information by adaptively weighting the hidden feature.

In order to fuse the obtained representations, the output features of the CNN-TCAM network and the GRU-Attention network are concatenated, and a fatten layer is utilized to reshape data into one-dimensional data suitable for the dense layer. The dense layer generates the prediction results. 


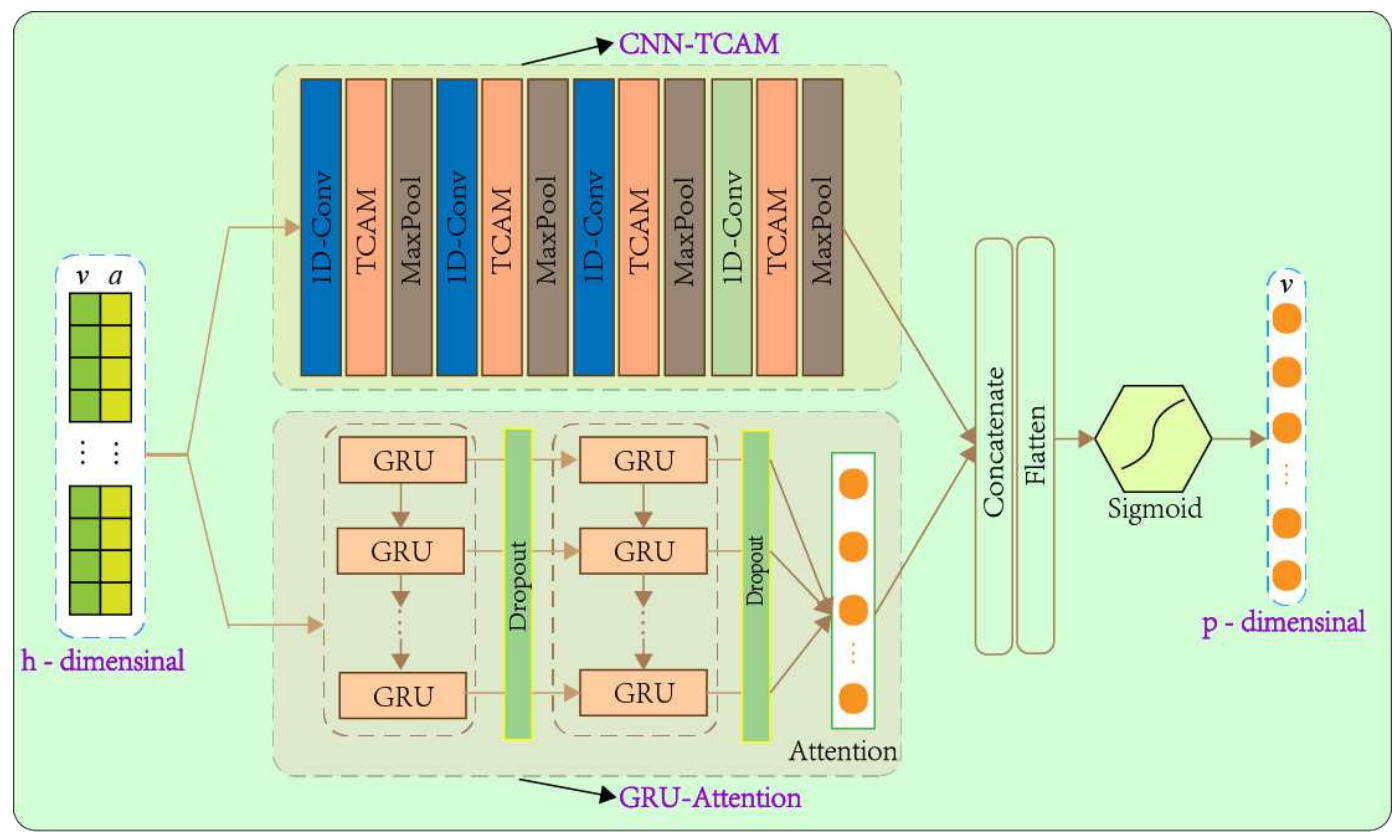

Figure 1. The architecture of our CGAM network

TCAM block. It would be great to make the network more sensitive to higher contribution features and focus on learning more essential features, which would enhance the network's representational power and improve its performance. So, keep that in mind and be inspired by ${ }^{30},{ }^{31}$, we design a temporal attention (TA) unit and a channel attention (CA) unit by exploiting the temporal and channel interdependencies of features, and we combine both attention types to modulate feature representations.

In the inputs and feature maps, the information is diverse along with the temporal locations. For example, the degree of impact of vehicle driving state at different historical moments on future speed is diverse. Therefore, we adopt a form of attention termed TA, which is helpful to make the network have the discriminative ability for different local regions and pay more attention to the more important regions. As shown in Figure 2, let $V=\left[v_{1}, v_{2}, \cdots v_{C}\right]$ be an input to the TA unit, which has $C$ feature maps with size of $N$. To generate temporal attention mask $\alpha$ with a size of $N$, we adopt two layers of convolutional neural network followed by a Sigmoid function and Softmax function. The definition of TA unit is as follow:

$$
\alpha=\operatorname{Softmax}\left(\sigma\left(W_{T A}^{2} * \delta\left(W_{T A}^{1} * V+b_{T A}^{1}\right)+b_{T A}^{2}\right)\right)
$$

where the meanings of the notations $\sigma(\cdot), \delta(\cdot)$ represent the sigmoid and SELU functions respectively, and $*$ denotes the convolution operation. $W_{T A}^{1}, b_{T A}^{1}, W_{T A}^{2}$ and $b_{T A}^{2}$ are the weights and bias in the first convolutional layer and the second convolutional layer. In addition, the temporal attention weights are adapted to the values between 0 and 1 by the sigmoid function $\sigma(\cdot)$, and then with the normalization of Softmax function used for normalization to ensure the limited additivity of weights. The attention weights $\alpha$ is utilized to rescale the input features as follows:

$$
\Phi_{T A}(V)=f_{T A}(V, \alpha)
$$

where $f_{T A}(\cdot)$ is an element multiplication for temporal positions of each feature map and their corresponding spatial attention weights, and the TA unit is denoted by $\Phi_{T A}(\cdot)$.

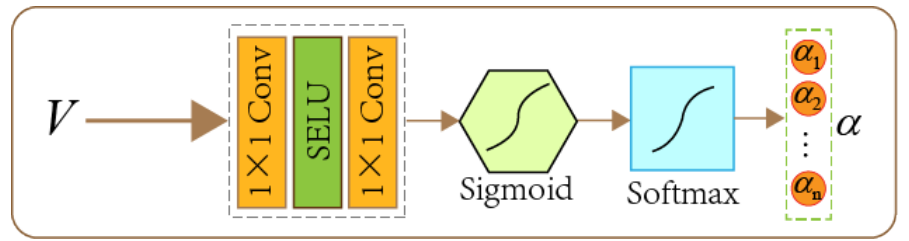

Figure 2. The operations of temporal attention

On the other hand, each feature map channel can be considered a feature detector, and there is redundant information in 
feature maps. Therefore, the objective of the CA unit is to emphasize informative feature maps while suppressing irrelevant ones selectively. The operations of the channel are shown in Figure 3.

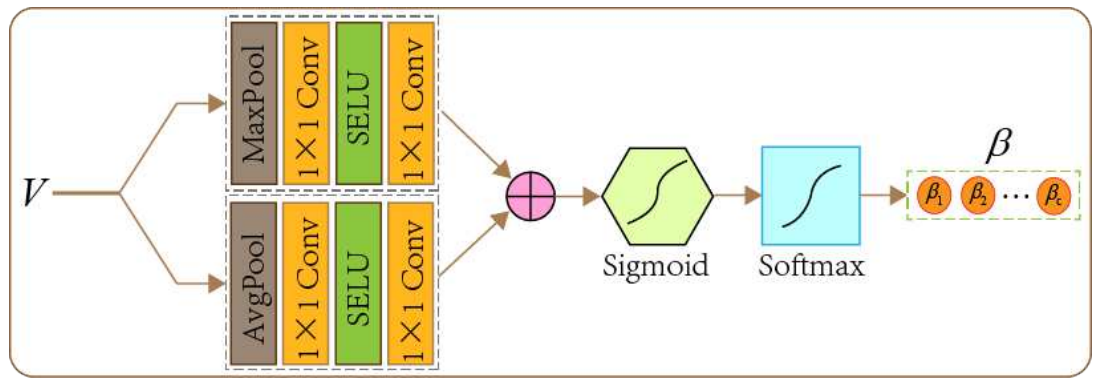

Figure 3. The operations of channel attention

The channel attention process produces a channel attention map by analyzing the features that are interrelated between channels. We squeeze the temporal dimension of the input feature map to compute the channel attention efficiently. For aggregating temporal information, both the max-pooling and the global average-pooling are operated on individual feature channels along temporal dimensions, generating two different channel context descriptors $Z_{c}^{\max }$ and $Z_{c}^{a v g}$, which denote maxpooled features and average-pooled features, respectively. Both descriptors are then forwarded to convolutional network respectively to produce our channel attention map $M_{c}^{\max }$ and $M_{c}^{a v g}$. After applying the convolutional layer to each descriptor, we merge the output feature vectors. We describe the detailed operation using formulas as below:

$$
\begin{gathered}
z_{c}^{\max }=\operatorname{MaxPool}\left(v_{c}\right) \\
z_{c}^{a v g}=\operatorname{AvgPool}\left(v_{c}\right) \\
M_{C A}^{\max }=W_{\max }^{2} * \delta\left(W_{\max }^{1} * Z_{c}^{\max }+b_{\text {max }}^{1}\right)+b_{\text {max }}^{2} \\
M_{C A}^{a v g}=W_{\text {avg }}^{2} * \delta\left(W_{\text {avg }}^{1} * Z_{c}^{a v g}+b_{\text {avg }}^{1}\right)+b_{\text {avg }}^{2} \\
\beta=\operatorname{Softmax}\left(\sigma\left(M_{T C}^{\max }+M_{T C}^{\text {avg }}\right)\right)
\end{gathered}
$$

where $\sigma(\cdot), \delta(\cdot)$ and $*$ represent the same operation as those used in Eq1. $W_{\text {max }}^{1}, W_{\text {max }}^{2}, W_{\text {avg }}^{1}$ and $W_{\text {avg }}^{2}$ are the weight parameters of the convolutional operation. $b_{\text {max }}^{1}, b_{\text {max }}^{2}, b_{a v g}^{1}$ and $b_{a v g}^{2}$ are the bias parameters of the convolutional operation. Further, the channel attentive map mask $\beta$ to be obtained by the sigmoid function and the Softmax function. The sum of all the components of the attentive map is $1 . V_{C A}$ is the output of the CA unit $\Phi_{C A}(\cdot)$, and it can be produced as follow:

$$
\Phi_{C A}(V)=f_{C A}(V, \beta)
$$

where $f_{C A}(\cdot)$ is a channel-wise multiplication for feature channels and corresponding channel weights.

With the help of the TA unit and CA unit, the importance of input features is automatically modulated to enhance the representational power of the network. Inspired by the residual network introduced $\mathrm{in}^{32}$, it can improve information flow and achieve better performance for NNs. We apply the combinational attention model $\Phi(\cdot)$ to modulate the residual feature map $V$. We integrate the TA unit and CA unit into the residual network. The residual feature map $V$ is fed to TA unit $\Phi_{T A}(\cdot)$ and CA unit $\Phi_{C A}(\cdot)$ respectively, hence we can obtain two sets of temporal weighted feature maps $V_{T A}$ and channel weighted feature maps $V_{C A}$. Next, in order to fuse two sets of modulated feature maps, $V_{T A}$ and $V_{C A}$ are concatenated as the input to a convolutional layer. The processes can be formulated as follows.

$$
\begin{gathered}
V_{T A}=\Phi_{T A}(V) \\
V_{C A}=\Phi_{C A}(V) \\
\Phi(V)=W_{\Phi} *\left[V_{T A}, V_{C A}\right]+b_{\Phi}
\end{gathered}
$$

where $[\cdot]$ represents the operation of feature concatenation. The overall structure of the TCAM block is shown in Figure 4.

$M_{I}$ and $M_{O}$ express as the input and output of a TCAM block, and if we denote $\Phi(\cdot)$ as the combinational attention model of TA unit and CA unit, the TCAM block can be expressed as follows:

$$
M_{O}=\mathscr{F}\left(M_{I}\right)=M_{I}+\Phi(V)=M_{I}+\Phi\left(\mathscr{R}\left(M_{I}\right)\right)
$$




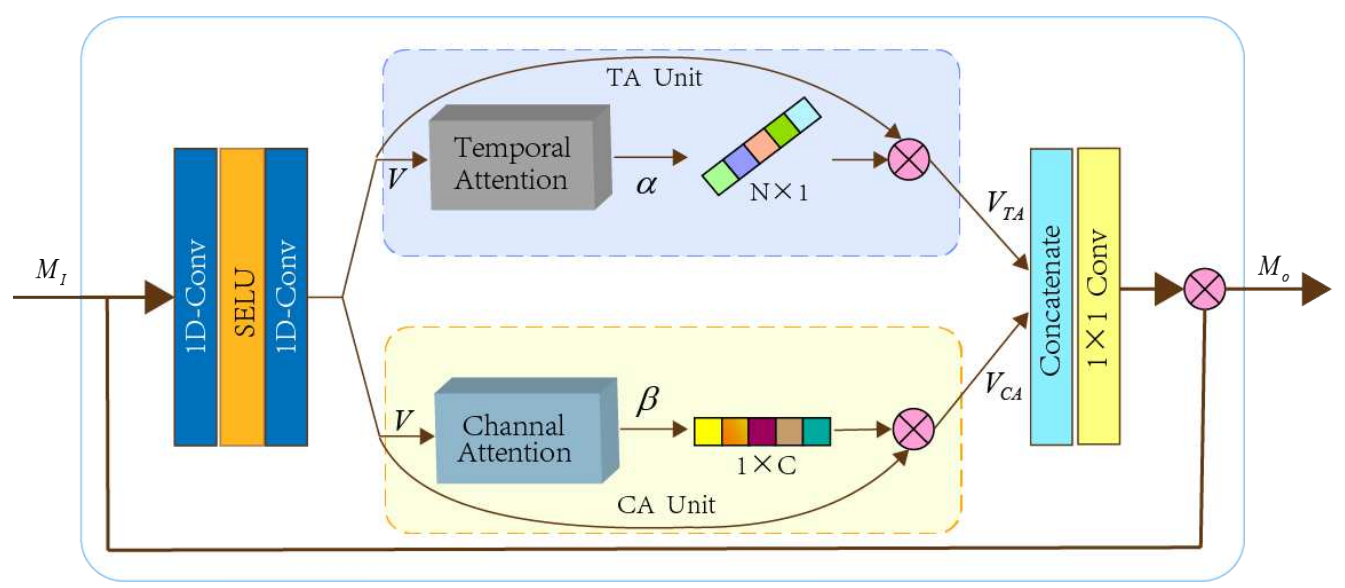

Figure 4. Temporal and channel attention model(TCAM)

$$
\mathscr{R}\left(M_{I}\right)=W_{\mathscr{R}}^{2} * \delta\left(W_{\mathscr{R}}^{1} * M_{I}+b_{\mathscr{R}}^{1}\right)+b_{\mathscr{R}}^{2}
$$

where $\mathscr{F}(\cdot)$ and $\mathscr{R}(\cdot)$ represent the functions of the TCAM block and the residual branch, respectively. There are two stacked convolutional layers with a SELU activation in the residual branch. $W_{\mathscr{R}}^{1}, W_{\mathscr{R}}^{2}, b_{\mathscr{R}}^{2}$ and $b_{\mathscr{R}}^{1}$ are the weight and bias in the convolutional layer of the residual branch.

The TCAM block has the excellent property of mapping and can capture more critical information. It inserted the TCAM block into convolutional networks to help the network boost its feature representational power.

\section{Results}

This part intends to show implementation details and the results of our experiments to assess the proposed method.

Data. Vehicle speed prediction by collecting vehicle driving data for model training and learning. In order to collect operation data, a typical operation scenario is first constructed in the IPG-CarMaker vehicle operation virtual simulation environment. Its graphical user interface (GUI) is shown in Figure 5.

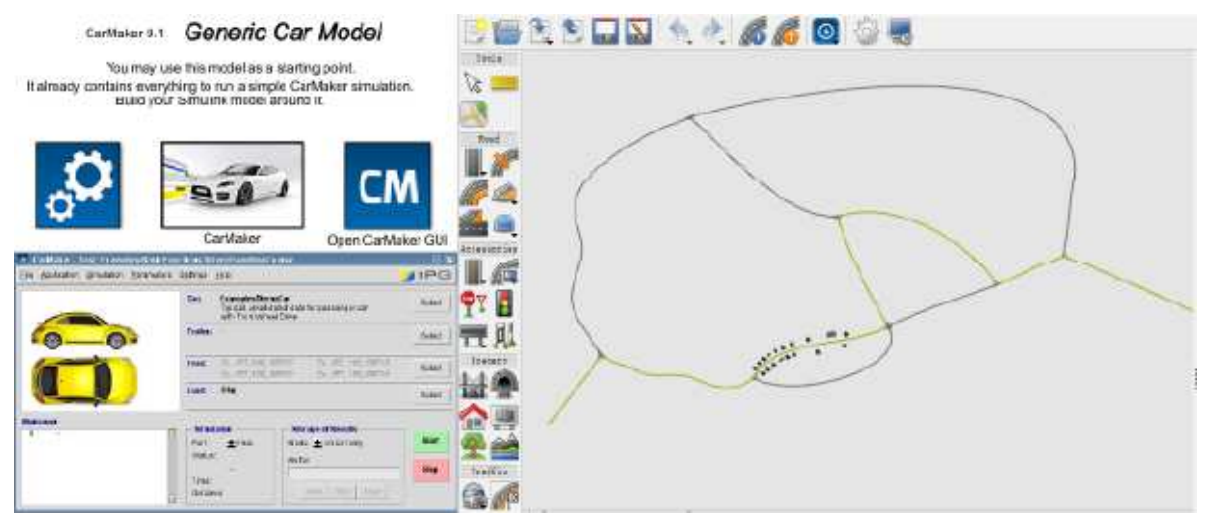

Figure 5. GUI of traffic simulation platform

The scenario model includes the road environment, the configuration of the vehicle model, the traffic lights, and the traffic flow settings. The road passes through six-light traffic intersections throughout, the vehicle's driving speed is not allowed to exceed $80 \mathrm{~km} / \mathrm{h}$, and the road traffic flow is random. It should be noted that even in the same scenario, the data of each vehicle operation is different due to the influence of the vehicle and traffic environment. Therefore, the simulation data can be used for training learning of the prediction model. The speed profile of the simulated vehicle for one driving cycle is shown in Figure 6.

The sampling time of vehicle speed in the simulation environment is 0.05 seconds in original depending on the communication protocol among different vehicle controllers. Considering the accuracy of prediction and the complexity of comprehensive calculation, a sampling time of 1 second is finally adopted ${ }^{18}$. Unprocessed data are not conducive to training neural networks. Data standardization involves scaling the data to a specific range. In this study, the MaxAbsScaler method is used to preprocess the data. The normalization method increases the gradient descent speed and improves the prediction accuracy. 


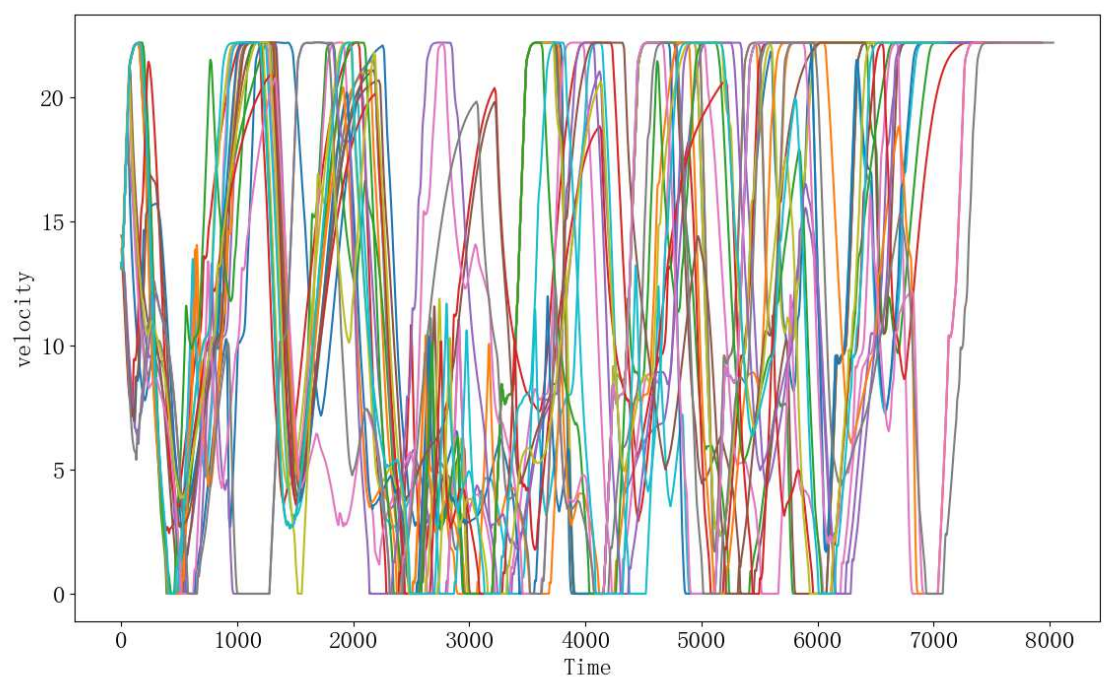

Figure 6. A part of the collected speed profile

Instance creation. In the vehicle speed prediction task, h-seconds historical speed data is used to predict the p-seconds future vehicle velocity. A sliding window which continuously slides over the dataset with one step is utilized to Split historical data. The input sample matrix $X$ is formed as $\left[x_{1}, x_{2}, \cdots x_{h}\right]^{T}$, and the label sample matrix $\left[y_{1}, y_{2}, \cdots y_{p}\right]^{T}$. The construction of instances is depicted in Figure 7.

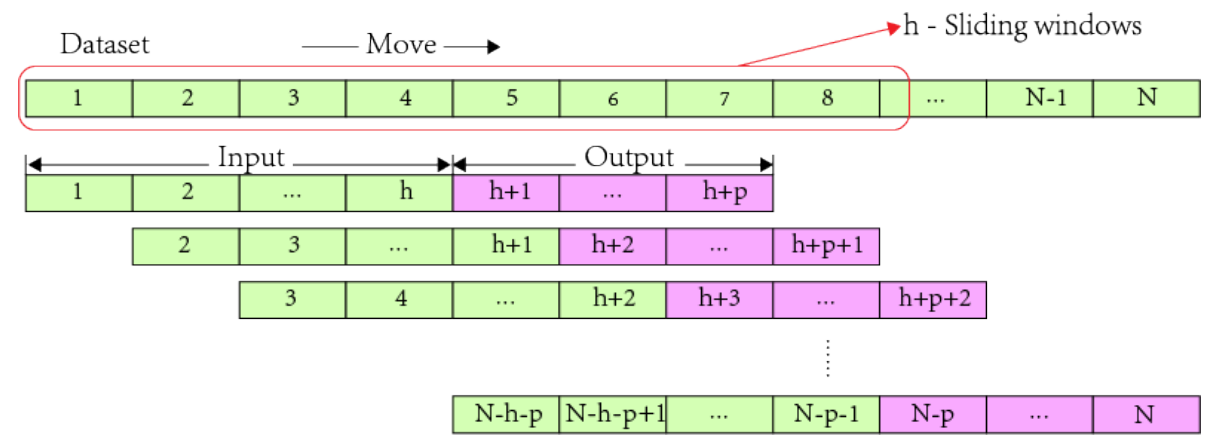

Figure 7. Instance creation for a prediction model

Performance metrics. Mean Absolute Error (MAE), Root Mean Square Error (RMSE), and Determination Coefficient (R2), defined as the following forms, are adopted as evaluation metrics to the performance of the prediction model in experiments.

$$
\begin{array}{r}
M A E=\frac{1}{n} \sum_{i=1}^{n}\left|y_{i}-\widehat{y}_{i}\right| \\
R M S E=\sqrt{\frac{1}{n} \sum_{i=1}^{n}\left(y_{i}-\widehat{y}_{i}\right)^{2}} \\
R^{2}=1-\frac{\sum_{i=1}^{n}\left(y_{i}-\widehat{y}\right)^{2}}{\sum_{i=1}^{n}\left(y_{i}-\bar{y}_{i}\right)^{2}}
\end{array}
$$

where $y_{i}$ denotes the real value, $\widehat{y}_{i}$ represents the forecast value, $\bar{y}_{i}$ is the average value of the real value sequence, and $n$ means the number of test samples. 
RMSE and MAE evaluate the accuracy of regression results. The smaller the values of RMSE and MAE, the model's prediction accuracy is the more accurate. R2 is an index to evaluate the fitting quality of the prediction model, which measures the fitting degree of the predicted value to the actual value. The bigger the value of $R^{2}$, the better the fitting extent of the prediction model.

Experimental setup. This paper implements the model in Window10 operating system, AMD Ryzen $73700 \mathrm{X} 8 *$ core, 3.6 GHz CPU, 16GB memory, and NVIDIA GTX 1080Ti platform. The programming language is python3, and the deep learning frameworks are Keras and Tensorflow2.0.

To fully evaluate the performance of the proposed CGAM model and the CNN-TCAM model, other existing five reference models, CNN, GRU, LSTM, GRU with Attention (GRU-ATT), LSTM with Attention (LSTM-ATT), are also built for comparisons. The training is carried out in mini-batches with a batch size of 128 , and all the models are trained for 250 epochs. In order to avoid the overfitting problem, dropout is widely used between layers except for convolution layers with the probability of 0.5 . If the loss of the past epoch is greater than that of the current epoch of the validation data set, the trained model will be saved as the best model. Furthermore, all models used an early stopping condition during the training, which stops the training if the validation loss on the validation data does not change within 15 training epochs. Adam is chosen as the optimizer of these models.

Too many neural network layers will increase the network parameters and complexity; too few neural network layers will lead to insufficient feature extraction. In this experiment, the network structures are set as follows.

CNN: Four convolution layers with max-pooling are stacked. They use $[32,64,64,32]$ filters respectively with size $1 \times 3$. All the activation functions in the model are SELU.

GRU and LSTM: The number of GRU and LSTM layers is fixed by 2. We set the same size of hidden states for each layer, that is, hidden-size is 32, and tanh activation function is used.

GRU-ATT or LSTM-ATT: A consequence of using an attention mechanism is integrating information over time. Consequently, we first feed the GRU or LSTM outputs through a one-layer Muti-Layer Perception (MLP) to obtain hidden representations, then calculate the normalized importance weights, thus further computing the feature outputs which can be utilized to predict vehicle speed.

CNN-TCAM: TCAM module is embedded into the CNN network. The network structure of the TCAM module is elaborated in the TCAM Block.

CGAM Network: We apply a combinational method which can connect CNN-TCAM and GRU-ATT to structure CGAM Network, two sets of modulated feature maps are concatenated as a prediction feature map.

Simulation results and discussion. Under the same simulation experimental conditions, we compare the proposed prediction models and the other five reference models.

We analyzed the effects of different lengths of historical input information on the prediction results. Figure 8 shows the prediction results of vehicle speed in the next 10 seconds under historical input information of two different lengths. In order to more accurately represent the performance of different prediction models, Table 1 shows the prediction results of vehicle speed at the 5th and 10th seconds when the lengths of historical input information are 10 seconds and 30 seconds, respectively.

Table 1. Performance metric comparison of prediction from different models

\begin{tabular}{clcc|cc|cc}
\hline \hline \multirow{2}{*}{ Input_length } & Forecasting method & \multicolumn{3}{c}{ Performance metric } \\
\cline { 3 - 7 } & & \multicolumn{2}{c}{ MAE } & \multicolumn{2}{c}{ RMSE } & \multicolumn{2}{c}{ R2 } \\
\cline { 2 - 7 } & CNN & 1.591 & 2.998 & 2.170 & 4.086 & 0.919 & 0.721 \\
& CNN-TCAM(ours) & 1.218 & 2.383 & $\mathbf{1 . 9 4 8}$ & 3.591 & $\mathbf{0 . 9 3 5}$ & 0.785 \\
& GRU & 1.708 & 2.885 & 2.347 & 3.926 & 0.905 & 0.742 \\
& GRU-ATT & 1.690 & 2.883 & 2.304 & 3.956 & 0.909 & 0.739 \\
& LSTM & 1.582 & 2.816 & 2.252 & 3.925 & 0.912 & 0.743 \\
& LSTM-ATT & 1.541 & 2.797 & 2.187 & 3.899 & 0.917 & 0.746 \\
& CGAM(ours) & $\mathbf{1 . 2 1 1}$ & $\mathbf{2 . 3 5 9}$ & 1.966 & $\mathbf{3 . 5 8 1}$ & 0.933 & $\mathbf{0 . 7 8 6}$ \\
\hline \multirow{3}{*}{$30 \mathrm{~s}$} & 1.409 & 2.385 & 1.987 & 3.260 & 0.933 & 0.828 \\
& CNN & 1.236 & 2.080 & 1.951 & 3.057 & 0.936 & 0.841 \\
& CNN-TCAM(ours) & 1.396 & 2.285 & 1.974 & 3.220 & 0.934 & 0.825 \\
& GRU & 1.424 & 2.248 & 1.951 & 3.123 & 0.935 & 0.831 \\
& GRU-ATT & 1.328 & 2.159 & 1.906 & 3.143 & 0.938 & 0.848 \\
& LSTM & 1.346 & 2.079 & 1.921 & 3.091 & 0.937 & 0.838 \\
& LSTM-ATT & $\mathbf{1 . 0 9 9}$ & $\mathbf{1 . 8 9 7}$ & $\mathbf{1 . 7 5 4}$ & $\mathbf{2 . 9 2 1}$ & $\mathbf{0 . 9 4 8}$ & $\mathbf{0 . 8 5 3}$ \\
\hline \hline
\end{tabular}



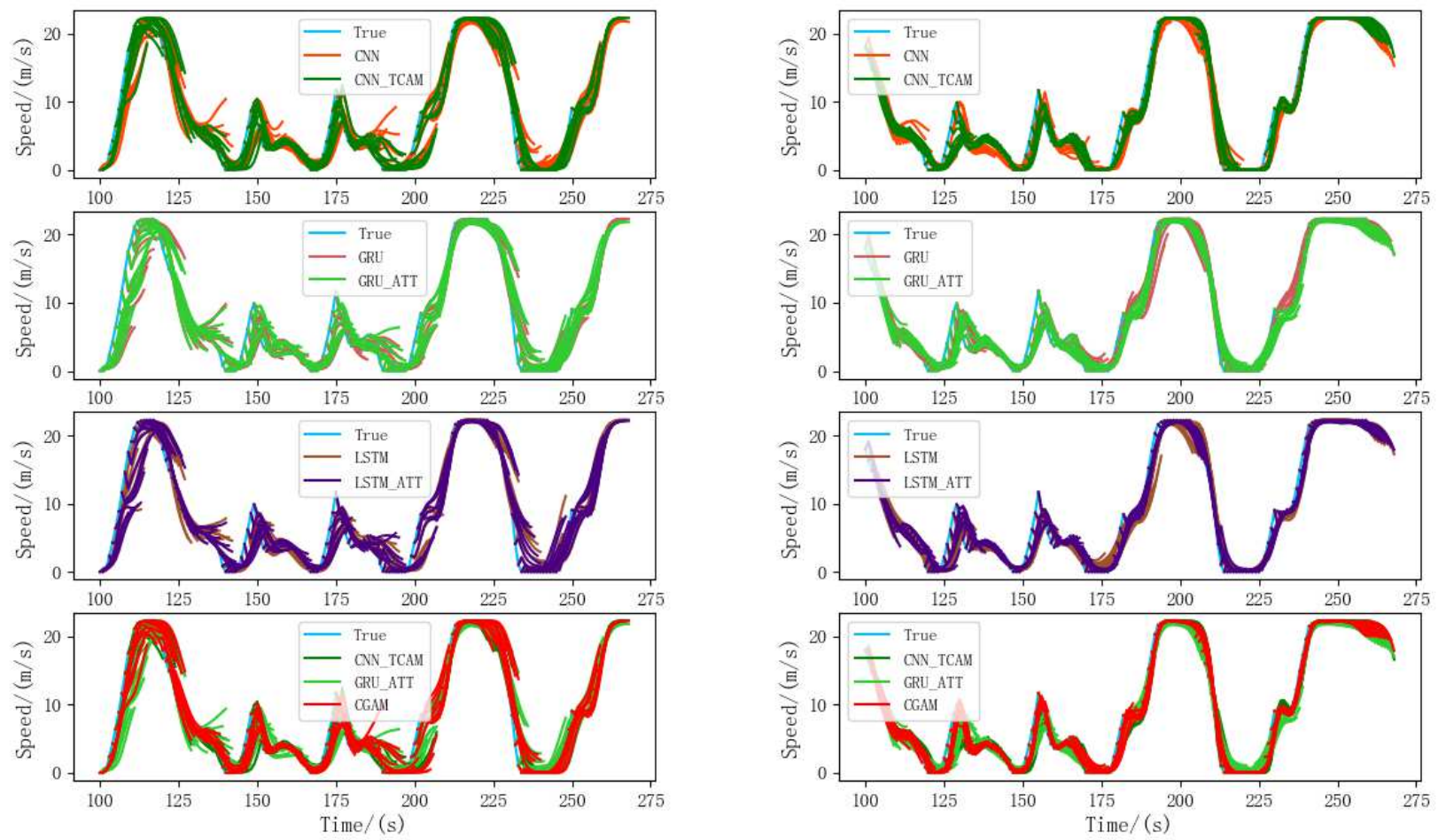

(a) The input length of historical information 10 seconds

(b) The input length of historical information 30 seconds

Figure 8. Speed prediction results in the next 10 seconds under historical input information of two different lengths
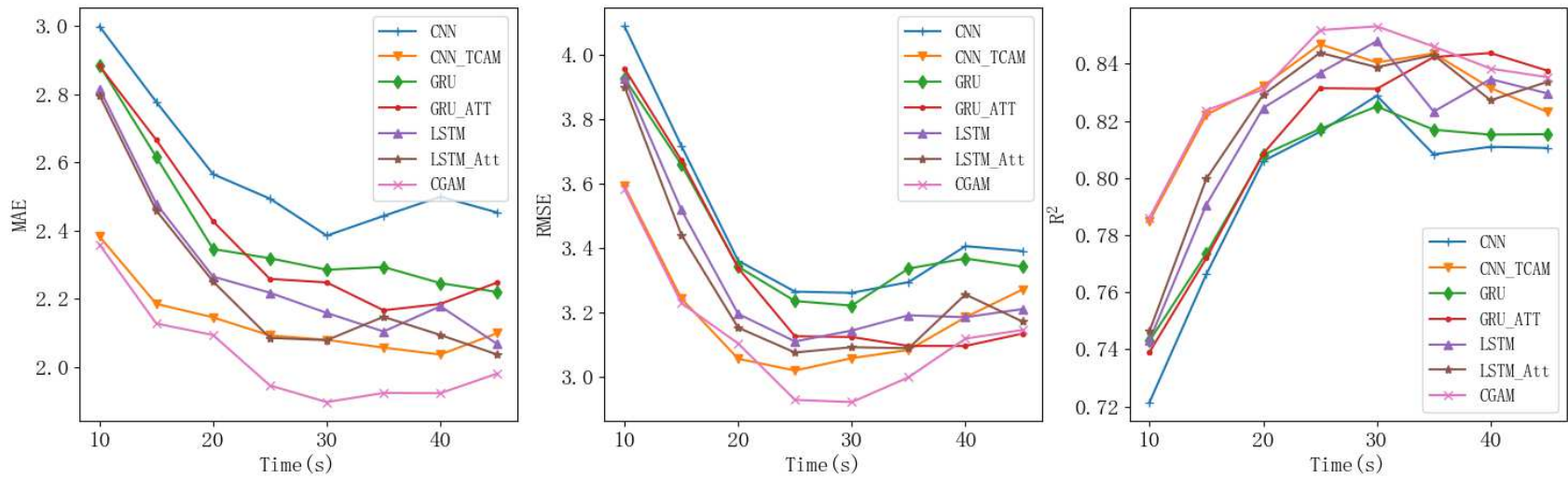

Figure 9. Performance metrics comparison of prediction from different models which the lengths of historical input information are between 10 and 45 seconds with an interval of 5 seconds

Figure 8 and Table 1 show the impact of historical data length on forecasting performance. When the input information length is 30 seconds, the prediction performance of all models is significantly better than that when the input information length is 10 seconds. The information provided by the input feature, which is too short, is no longer enough, and the training model will not learn the mapping relationship between historical driving information and future vehicle speed, leading to prediction error. Meanwhile, as shown in Figure 9, the 10th-second prediction results of models in which the lengths of historical input 

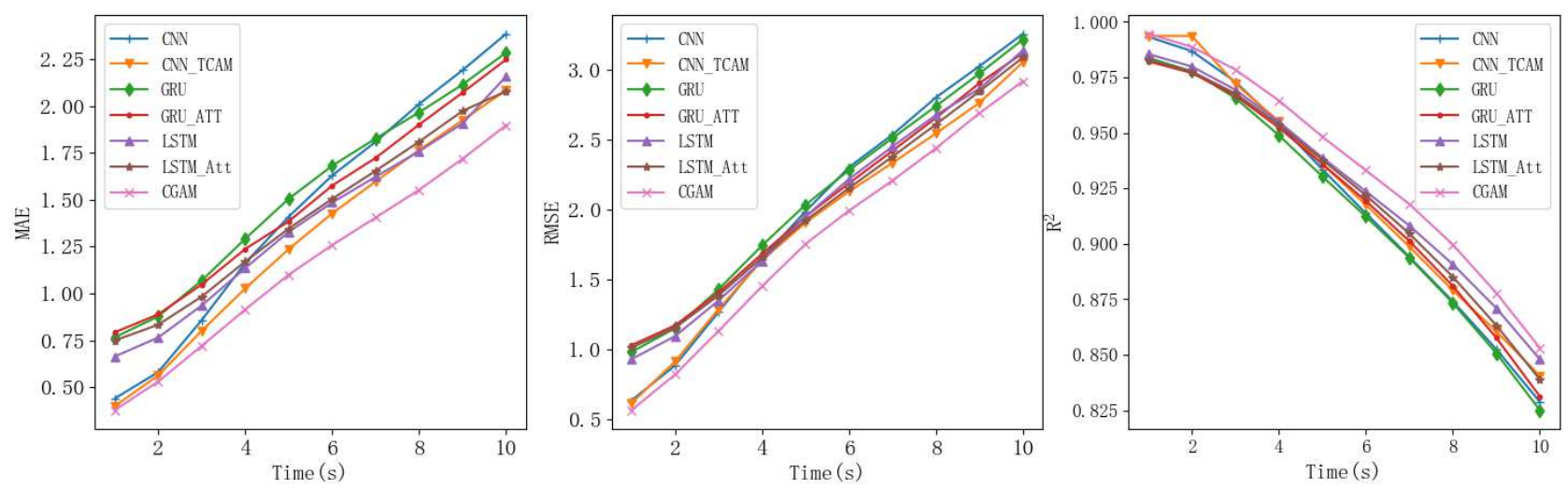

Figure 10. Speed prediction performances for the next 10 seconds with the length of history input information 30 seconds

information are between 10 and 45 seconds with an interval of 5 seconds are compared. As the length of historical information increases, the forecasting performances of models are improving. However, errors increase to different degrees for speed prediction performance in which the input sequence exceeds the 35s horizon. Over-fitting is likely to occur if the time-step of input data is too long. By comparing different evaluation metrics, the results show that the CGAM model and the CNN-TCAM mode outperform other baselines in most cases.

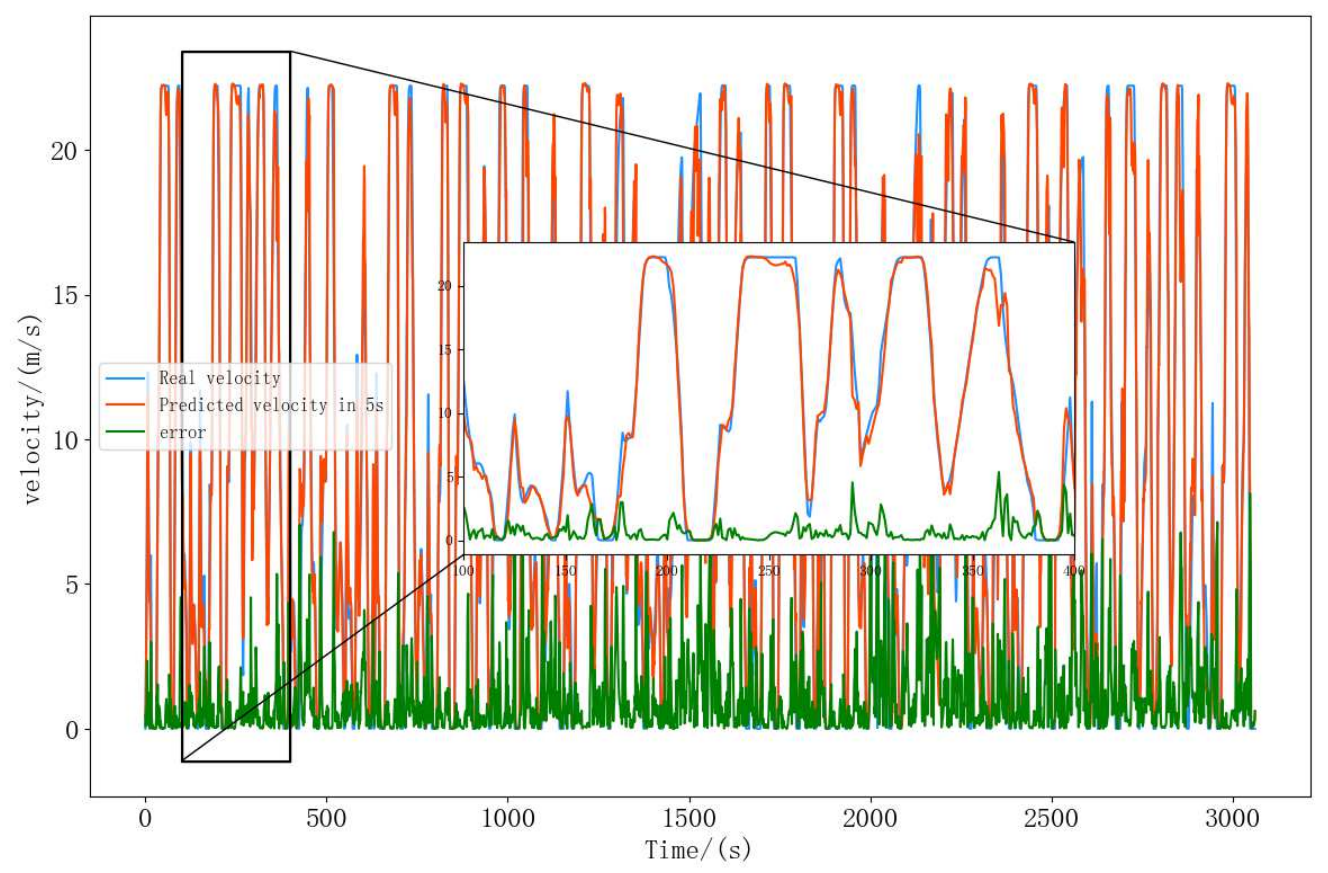

Figure 11. The results of prediction curve and absolute error curve at the 5th second using the CGAM network that the lengths of historical input information is 30 seconds.

The benefits of attention methods are analyzed as follows. According to the comparison of prediction of different models from the Figure8 and the Table 1, the proposed CNN-TCAM model performed better than the CNN model mostly. The results suggest TCAM Block can apply temporal attention and channel attention to capture more critical information. According to Figure 9, the forecasting performances of the GRU and GRU-ATT models are similar when the input sequence length is 
less than 25 steps. Besides, the GRU-ATT model outperforms the GRU model most times. Therefore, attention methods can strengthen the feature extraction ability of the original GRU model.

Analyzing the model error can help us determine the direction for improving the accuracy of predictions. As shown in Figure 8, it can be seen that the profiles of predicted speed reflect the changing trend of vehicle driving speed in the future. Figure 10 shows the prediction performances of speed forecast for the next 10 seconds when the length of history input information is 30 seconds. The errors show an evident temporal variation trend that the prediction error of each model will increase with time. Because of the imperfect forecasting architecture and input information, models lose accuracy gradually.

Figure 11 shows the results of the prediction curve and absolute error curve at the 5th second using the CGAM network that the lengths of historical input information are 30 seconds. The Orange line represents the predicted future speed, and the green line is the absolute error curve between actual speed and predicted speed. We can see that the error will become larger when the vehicle stops and starts. For where the acceleration changes considerably, the vehicle speed prediction errors will also increase.

\section{Conclusion and future works}

This paper proposes a novel vehicle speed prediction model CGAM based on the CNN and GRU model with an attention mechanism. CNN, GRU, LSTM, GRU-ATT, LSTM-ATT, CNN-TCAM, CGAM are evaluated in the experiment. Under the verification of MAE, RMSE, R2, the proposed CGAM model used in the experiment performed better than other models. Therefore, the CGAM network can better deal with vehicle speed prediction. Comparing the CNN and CNN-TCAM models reflects the benefits of the proposed TCAM Block, which has the excellent property of boosting the network representational power. In recurrent neural networks, attention mechanisms can make full use of important input information that helps the networks improve prediction accuracy in most cases. Inspired by the Simulation results and discussion section, our following research will consider further improving the model's performance by reducing the error where the speed changes significantly and utilizing the information provided through vehicle-to-vehicle (V2V) and vehicle-to-infrastructure (V2I) communication channels.

\section{Data availability}

The datasets used and/or analysed during the current study available from the corresponding author on reasonable request. The author can be contacted via email:2540590016@qq.com

\section{References}

1. Park, J. et al. Intelligent trip modeling for the prediction of an origin-destination traveling speed profile. IEEE Transactions on Intell. Transp. Syst. 15, 1039-1053 (2014).

2. Sun, C., Hu, X., Moura, S. J. \& Sun, F. Velocity predictors for predictive energy management in hybrid electric vehicles. IEEE Transactions on Control. Syst. Technol. 23, 1197-1204 (2014).

3. Cela, A. et al. Energy optimal real-time navigation system. IEEE Intell. Transp. Syst. Mag. 6, 66-79 (2014).

4. Butakov, V. A. \& Ioannou, P. Personalized driver assistance for signalized intersections using v2i communication. IEEE Transactions on Intell. Transp. Syst. 17, 1910-1919 (2016).

5. Chen, Y., Lu, C. \& Chu, W. A cooperative driving strategy based on velocity prediction for connected vehicles with robust path-following control. IEEE Internet Things J. 7, 3822-3832 (2020).

6. Liu, K., Asher, Z., Gong, X., Huang, M. \& Kolmanovsky, I. Vehicle velocity prediction and energy management strategy part 1: Deterministic and stochastic vehicle velocity prediction using machine learning. Tech. Rep., SAE Technical Paper (2019).

7. Shin, J. \& Sunwoo, M. Vehicle speed prediction using a markov chain with speed constraints. IEEE Transactions on Intell. Transp. Syst. 20, 3201-3211 (2018).

8. Deo, N., Rangesh, A. \& Trivedi, M. M. How would surround vehicles move? a unified framework for maneuver classification and motion prediction. IEEE Transactions on Intell. Veh. 3, 129-140 (2018).

9. Chen, Y., Hu, C. \& Wang, J. Motion planning with velocity prediction and composite nonlinear feedback tracking control for lane-change strategy of autonomous vehicles. IEEE Transactions on Intell. Veh. 5, 63-74 (2019).

10. Lefèvre, S., Sun, C., Bajcsy, R. \& Laugier, C. Comparison of parametric and non-parametric approaches for vehicle speed prediction. In 2014 American Control Conference, 3494-3499 (IEEE, 2014).

11. Amini, M. H., Kargarian, A. \& Karabasoglu, O. Arima-based decoupled time series forecasting of electric vehicle charging demand for stochastic power system operation. Electr. Power Syst. Res. 140, 378-390 (2016). 
12. Guo, J., He, H. \& Sun, C. Arima-based road gradient and vehicle velocity prediction for hybrid electric vehicle energy management. IEEE Transactions on Veh. Technol. 68, 5309-5320 (2019).

13. Jing, J. et al. Vehicle speed prediction using a cooperative method of fuzzy markov model and auto-regressive model. In 2017 IEEE intelligent vehicles symposium (IV), 881-886 (IEEE, 2017).

14. Gmira, M., Gendreau, M., Lodi, A. \& Potvin, J.-Y. Travel speed prediction based on learning methods for home delivery. EURO J. on Transp. Logist. 9, 100006 (2020).

15. Zhou, Y., Ravey, A. \& Péra, M.-C. A survey on driving prediction techniques for predictive energy management of plug-in hybrid electric vehicles. J. Power Sources 412, 480-495 (2019).

16. Zhang, L., Liu, W. \& Qi, B. Combined prediction for vehicle speed with fixed route. Chin. J. Mech. Eng. 33, 1-13 (2020).

17. Liu, J., Chen, Y., Zhan, J. \& Shang, F. An on-line energy management strategy based on trip condition prediction for commuter plug-in hybrid electric vehicles. IEEE Transactions on Veh. Technol. 67, 3767-3781 (2018).

18. Xiang, C., Ding, F., Wang, W. \& He, W. Energy management of a dual-mode power-split hybrid electric vehicle based on velocity prediction and nonlinear model predictive control. Appl. energy 189, 640-653 (2017).

19. Hochreiter, S. \& Schmidhuber, J. Long short-term memory. Neural computation 9, 1735-1780 (1997).

20. Wang, Y., Liao, W. \& Chang, Y. Gated recurrent unit network-based short-term photovoltaic forecasting. Energies 11, 2163 (2018).

21. Abduljabbar, R. L., Dia, H. \& Tsai, P.-W. Development and evaluation of bidirectional lstm freeway traffic forecasting models using simulation data. Sci. reports 11, 1-16 (2021).

22. Che, Z., Purushotham, S., Cho, K., Sontag, D. \& Liu, Y. Recurrent neural networks for multivariate time series with missing values. Sci. reports 8, 1-12 (2018).

23. Gaikwad, T. D., Asher, Z. D., Liu, K., Huang, M. \& Kolmanovsky, I. Vehicle velocity prediction and energy management strategy part 2: Integration of machine learning vehicle velocity prediction with optimal energy management to improve fuel economy. Tech. Rep., SAE Technical Paper (2019).

24. Shen, P., Zhao, Z., Zhan, X., Li, J. \& Guo, Q. Optimal energy management strategy for a plug-in hybrid electric commercial vehicle based on velocity prediction. Energy 155, 838-852 (2018).

25. Zhang, L., Liu, W. \& Qi, B. Energy optimization of multi-mode coupling drive plug-in hybrid electric vehicles based on speed prediction. Energy 206, 118126 (2020).

26. Jiang, B. \& Fei, Y. Vehicle speed prediction by two-level data driven models in vehicular networks. IEEE Transactions on Intell. Transp. Syst. 18, 1793-1801 (2016).

27. Zhang, C. et al. Real-time optimization of energy management strategy for fuel cell vehicles using inflated 3d inception long short-term memory network-based speed prediction. IEEE Transactions on Veh. Technol. 70, 1190-1199 (2021).

28. Wang, Y., Zhang, D., Liu, Y., Dai, B. \& Lee, L. H. Enhancing transportation systems via deep learning: A survey. Transp. research part C: emerging technologies 99, 144-163 (2019).

29. Han, S., Zhang, F., Xi, J., Ren, Y. \& Xu, S. Short-term vehicle speed prediction based on convolutional bidirectional lstm networks. In 2019 IEEE Intelligent Transportation Systems Conference (ITSC), 4055-4060 (IEEE, 2019).

30. Woo, S., Park, J., Lee, J.-Y. \& Kweon, I. S. Cbam: Convolutional block attention module. In Proceedings of the European conference on computer vision (ECCV), 3-19 (2018).

31. Hu, Y., Li, J., Huang, Y. \& Gao, X. Channel-wise and spatial feature modulation network for single image super-resolution. IEEE Transactions on Circuits Syst. for Video Technol. 30, 3911-3927 (2019).

32. He, K., Zhang, X., Ren, S. \& Sun, J. Deep residual learning for image recognition. In Proceedings of the IEEE conference on computer vision and pattern recognition, 770-778 (2016).

\section{Acknowledgments}

This work was supported by the National Natural Science Foundation of China (No.61973265).

\section{Author contributions statement}

Z.N.W.: planning, conceptualisation, data collation, methodology, algorithm development and generation of results, drafting paper content, writing, reviewing and editing. X.J.H.: planning, conceptualisation, supervision and mentoring. Z.Z.:methodology, algorithm development and generation of results. 


\section{Competing interests}

Te authors declare no competing interests. 


\section{Supplementary Files}

This is a list of supplementary files associated with this preprint. Click to download.

- Data.zip 